\title{
Karyomorphological Studies in a Few Exotic Varieties of Genus Morus L.
}

\author{
B. N. Susheelamma, Jalaja S. Kumar, S. B. Dandin, \\ M. S. Jolly, K. Sengupta and R. Raju \\ Mulberry Breeding and Genetics Laboratory, \\ Central Sericultural Reasearch and Training Institute, \\ Mysore-570008, India
}

Accepted July 21, 1989

Among the genera of complex species which have defied the efforts of taxonomists and remained critical and confused, the genus Morus L. seems to have a place of its own owing to free cross fertilization among the species. The available population is highly heterogeneous, hence the classification on gross morphological characters alone has become difficult. The information in the field of cytology, karyomorphology, reproductive biology etc. is inadequate to seek the answer for some of the problems faced by the classical taxonomists. Reports of Tahara (1909), Osawa (1920) and Janaki Ammal (1948) confine only to the chromosome counts. Meiotic behaviour of a few cultivars have been reported by Datta (1954), Seki (1952), Das (1961) and Gill and Gupta (1974). The only report of karyomorphological studies is that of Kundu and Sharma (1976) in a few Indian cultivars. For most of the remaining species there is no information on karyomorphology. In the present studies an attempt has been made to elucidate the karyomorphological details in some important mulberry cultivars of exotic origin.

\section{Materials and methods}

Ten exotic varieties, namely Kosen, Kokuso-13, Kokuso-21, Kokuso-27, Roso and Rokokuyoso belonging to Morus latifolia Poir., Kenmochi, Ichihei and Mizusawa belonging to $M$. bombycis Koidz. and Chinapeking beloning to $M$. alba maintained in the mulberry germplasm of the Institute, were taken for the present study. Cuttings of these varieties were planted in pots for experimental use. Somatic preparations were made from excised root tips. Root tips were pretreated with aqueous solution of 0.002 molar 8-hydroxyquinoline for 3 hours at $10^{\circ} \mathrm{C}$ and fixed in 3:1 acetic acid: alcohol for about 4 hours. After washing in water the root tips were hydrolysed in $1 \mathrm{~N} \mathrm{HCl}$ for seven minutes at $60^{\circ} \mathrm{C}$ and stained in $1 \%$ aceto-orcein. Squash preparations were made in $45 \%$ acetic acid. Photomicrographs and drawings of good and clear plates were made immediately. To ascertain the chromosome number and their morphology, a number of preparatons were examined in each variety. Camera lucida drawings were made at table level and idiograms were drawn using suitable scale. Karyotype classification was made according to Leven et al. (1964) by taking arm ratio into account.

\section{Observations}

Details of the somatic chromosome number, ploidy level chromatin length and karyotype formula are presented in Table 1 . Of the cultivars studied, five are diploids $(2 n=28)$, three are aneuploids $(2 n=30)$ and two are triploids $(2 n=42)$. 
ๆ 8 กั

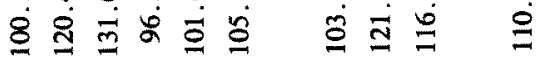

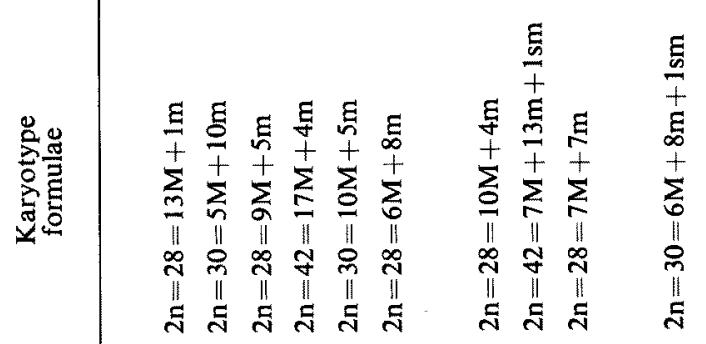

\section{$\stackrel{2}{\varrho}$}

辛

市市

$8 \stackrel{\infty}{\stackrel{\infty}{+} \stackrel{\infty}{+}} \stackrel{\infty}{+}$

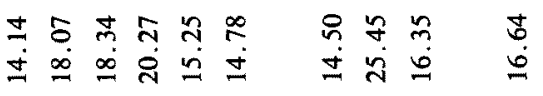

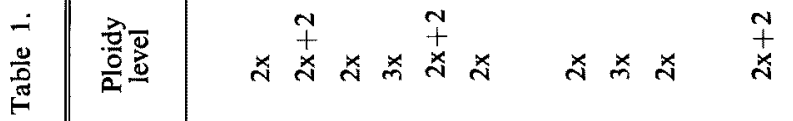

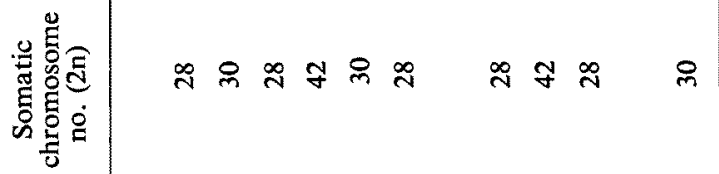

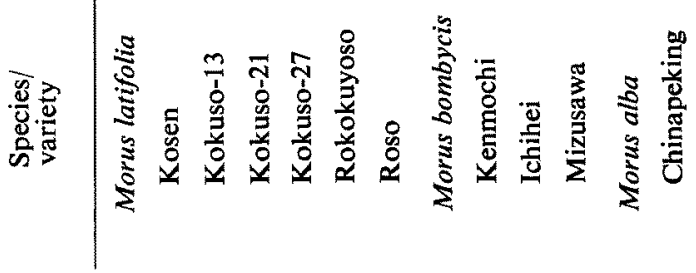


M. latifolia Poir. Cv. Kosen is one of the popular strains which was introduced to India from Japan in 1930's and propagated through grafts. Leaves are entire, large with good feeding quality. This is a diploid cultivar with $2 n=28$ chromosomes. Somatic chromosomes and idiograms are shown in Figs. $1-3(2 n=28=13 M+1 \mathrm{~m})$.

M. latifolia Poir. Cv. Kokuso-13 is a hybrid variety evolved by crossing Murasakiwase
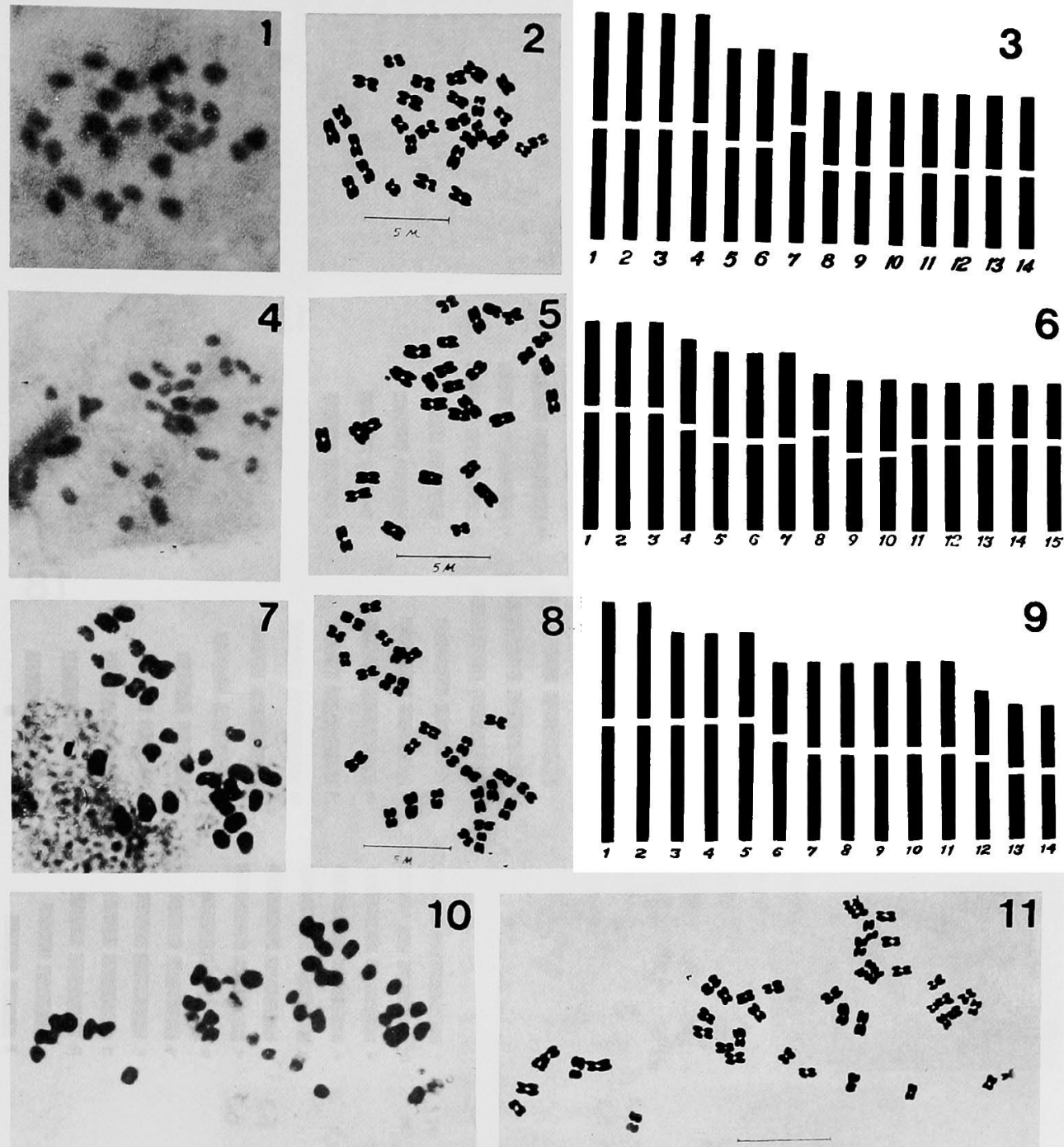

12

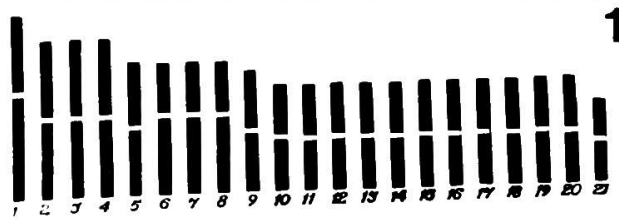

Figs. 1-12. 1-3, somatic chromosomes, camera lucida drawings and idiograms of Kosen $(2 n=28)$. $4-6$, somatic chromosomes, camera lucida drawings and idiograms of Kokuso-13 $(2 n=30)$. 7-9, somatic chromosomes, camera lucida drawings and idiograms of Kokuso-21 $(2 n=28)$. 10-12, somatic chromosomes, camera lucida drawings and idiograms of Kokuso-27 $(2 n=42)$. 
(M. bombycis) and Tsukasaguawa (M. latifolia). This bears lobed leaves with short internodes. Presence of $2 n=30$ chromosomes indicates the aneuploid nature of the cultivar $(2 n=30=$ $5 \mathrm{M}+10 \mathrm{~m}$ ). Somatic chromosomes and karyotype are shown in Figs. 4-6.

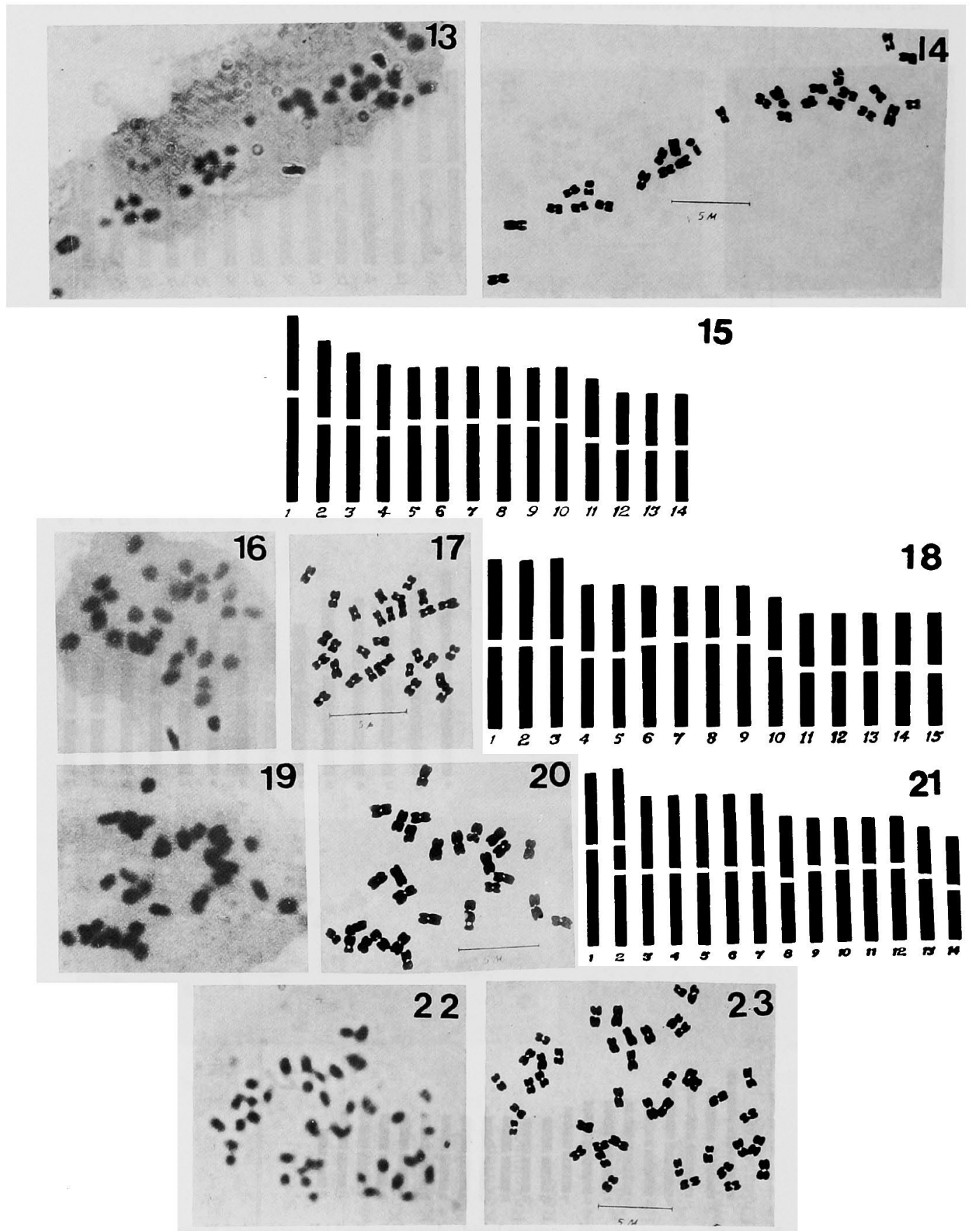

Figs. 13-23. 13-15, somatic chromosomes, camera lucida drawings and idiograms of Roso $(2 \mathrm{n}=28)$. 16-18, somatic chromosomes, camera lucida drawings and idiograms of Rokokuyso $(2 \mathrm{n}=$ 30). 19-21, somatic chromosomes, camera lucida drawings and idiograms of Kenmochi $(2 n=28)$. $22-23$, somatic chromosomes and camara lucida drawings of Ichihei $(2 n=42)$. 
M. latifolia Poir. Cv. Kokuso-21 is a diploid variety evolved by hybridization between Naganuma and Tsukasaguawa varieties of $M$. latifolia. This is recognised as the most popular

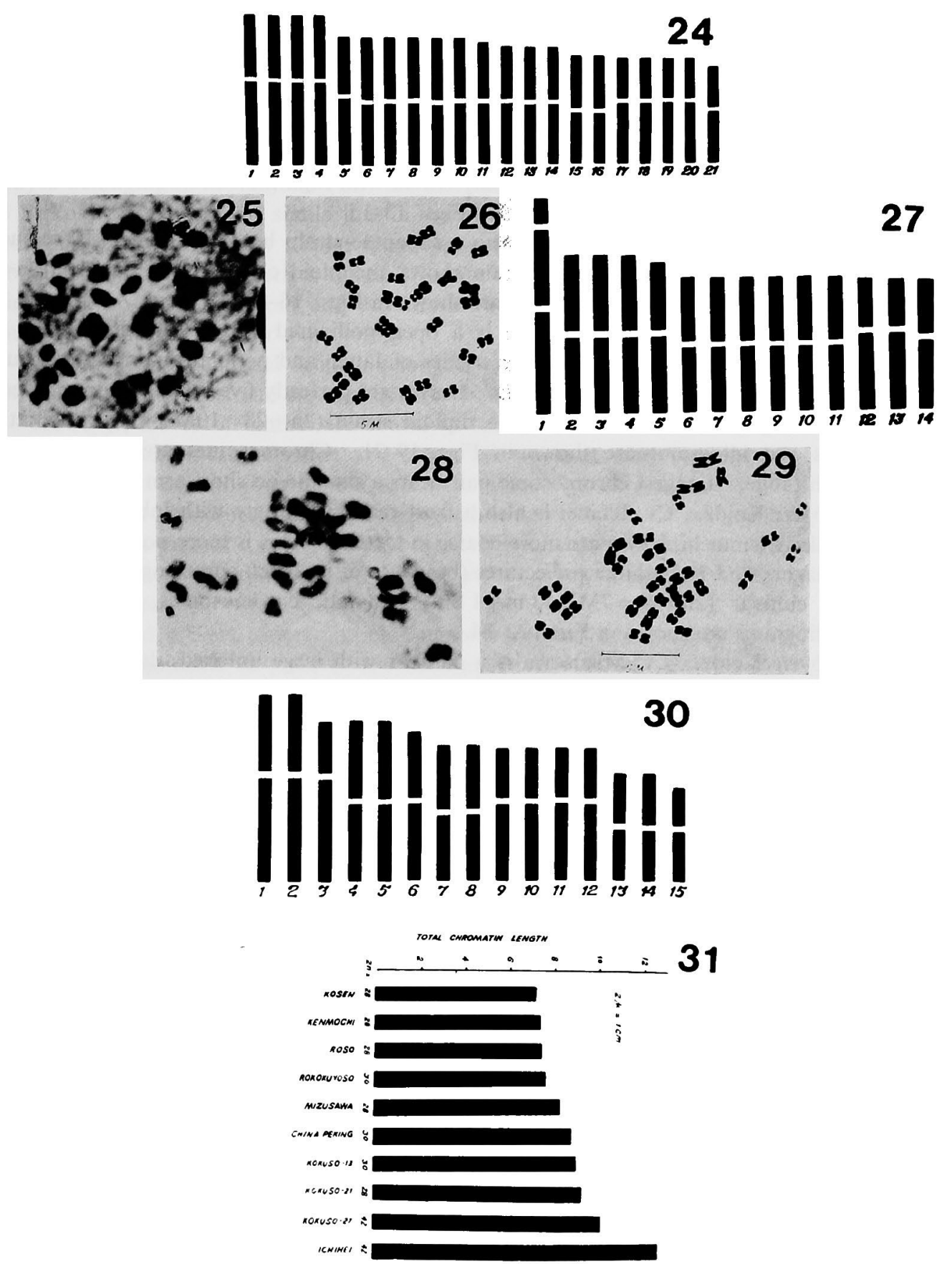

Figs. 24-31. 24, idiograms of Ichihei $(2 n=42)$. 25-27, somatic chromosomes, camera lucida drawings and idiograms of Mizusawa $(2 n=28)$. 28-30, somatic chromosomes, camera lucida drawings and idiograms of Chinapeking $(2 n=30) .31$, relative chromatin length of some exotic mulberry varieties. 
male component for interspecific crosses in Japan. Leaves are entire, glossy, deep green with smooth surface. Unlike the other two strains, the complement consists of two pairs of large chromosomes. Somatic chromosomes, camera lucida drawing and karyotypes are shown in Figs. $7-9(2 \mathrm{n}=28=9 \mathrm{M}+5 \mathrm{~m})$.

M. latifolia Poir. Cv. Kokuso-27 is a triploid cultivar $(2 n=42=17 M+4 m)$. This is a hybrid between unreduced gamete and reduced pollen of Naganuma ( $M$. latifolia) and Kairyonezumigaeki ( $M$. alba) which was released in the year 1948 (Seki 1952). Somatic chromosomes and idiograms are shown in Figs. 10-12.

$M$. latifolia Poir. Cv. Roso is a diploid variety of Japan $(2 n=28=6 M+8 m)$. This variety is characterised by large unlobed glossy leaves with high moisture content. Somatic chromosomes and idiograms are shown in Figs. 13-15.

$M$. latifolia Poir. Cv. Rokokuyso is also a Japanese strain bearing unlobed, large succulent and lustrous green leaves. This strain shows aneuploid chromosome number $(2 \mathrm{n}=$ $30=10 \mathrm{M}+5 \mathrm{~m}$ ). Karyotype and idiogram are shown in Figs. 16-18.

$M$. bombycis Koidz. Cv. Kenmochi is a open pollinated hybrid selection. This is one of the good rooting and frost resistant varieties of Japan and popular among the sericulturists of Tohoku and Hokuriku prefectures. Leaves are perfectly five lobed, medium sized, glossy and dark green in colour. This is a diploid strain $(2 n=28=10 M+4 m)$. Somatic chromosomes and idiograms are shown in Figs. 19-21. Chromosomes are small with a narrow length range. Longest chromosome pair bears a satellite on short arm.

$M$. bombycis Koidz. Cv. Ichihei is also a frost resistant variety with lobed dark green leaves. Unlike Kenmochi, leaves are more coarse in texture. This is more popular in Iwate, Miyagi, Yamagata and Fukushima prefectures (beacause of its quick sprouting nature). This is a triploid cultivar $(2 \mathrm{n}=42=7 \mathrm{M}+13 \mathrm{~m}+1 \mathrm{Sm})$. Somatic chromosomes, camera lucida sketch and idiograms are shown in Figs. 22-24.

$M$. bombycis Koidz. Cv. Mizusawa is a cultivar with large unlobed, dark green, thin leaves having longest petiole and is native of Japan. This is a diploid with 28 somatic chromosomes $(2 n=28=7 M+7 m)$. One pair is larger in size and the second largest pair bears satellite in short arm. (Figs. 25-27).

M. alba L. Cv. Chinapeking is a strain with variously lobed large leaves. Leaves are thin and characterised by round apecies and deeply cut incissions. This is also an aneuploid strain $(2 \mathrm{n}=30=6 \mathrm{M}+8 \mathrm{~m}+1 \mathrm{Sm})$. Karyotype and idiograms are shown in Figs. 28-30.

Comparative chromatin length of all ten cultivars is shown in Fig. 31.

\section{Discussion}

To classify existing mulberry populations objectively as taxa of different magnitude is rather difficult owing to the continuous variations as a result of out crossing. To evolve dependable system of classification, a study of all the four types of relationships viz. Phylogenetic, Phenotypic, Geotropic and Tropic (interdependent) is imperative as stated by Chennaveeraiah (1983). Clausen et al. (1936) considered that morphology, cytology and ecology of the taxa furnish critical data for studying phylogenetic evolution. Baker (1970) quotes Mayer (1942) that for determination of biological species in cultivated plants the observational bases are full description of morphological variation, physiological and biochemical information, chromosome number and karyotype analysis and evidence of natural hybridisation. Nakajima (1982, unpublished) supported this view and pointed out the paucity of information with regard to mulberry.

Koidzumi (1917) and Hotta (1953) have classified the genus Morus based on the morphological characters of female flower. Katsumata (1972) observed some anatomical char- 
acters in addition to morphological features. The above systems are considered to be incomplete as data from cytology, embryology etc. are lacking. Kundu and Sharma (1976) have analysed the karyotypes of a few mulberry cultivars belonging to $M$. alba L., M. mungolika Hort., $M$. multicaulis Perr. and $M$. nigra L. The other reports confine only to chromosome number (Tahara 1909, Sinoto 1929, Janaki Ammal 1948, Katsumata 1979). Gill and Gupta (1978) while studying cytology of sex types of Morus species reported the presence of large sized chromosomes. Datta (1954) and Das (1961) have studied meiosis in a few cultivars of $M$. alba, $M$. latifolia and $M$. indica.

In the present investigation, Kosen, Kokuso-13, Kokuso-21, Kokuso-27, Roso and Rokokuyso belonging to $M$. latifolia Poir. are morphologically distinct. Varieties, Roso, Kosen and Rokuyso, show some similarities in their thick glossy leaves with identical leaf margin. Cytologically also they show similar karyotype with only two types of chromosomes, equal chromatin length and also length range. Despite of common origin, Kokuso-13 is an aneuploid, Kokuso-21 is a diploid and Kokuso-27 is a triploid. This different chromosome number has reflected on their morphology.

Cultivars Kenmochi and Ichihei belonging to $M$. bombycis Koidz. have similarity in their adaptation i. e. frost and die-back resistance, lobed and dark green leaves, good rooting, etc. However, leaves of Ichihei are coarse in texture. Induction of triploidy has resulted in variation of chromosome shape and size. Mizusawa, another cultivar of M. bombycis, has large thin leaves with long petiole unlike the other two. Cytologically also it is different in having Sat chromosomes. Chinapeking which is aneuploid is the only cultivar of $M$. alba L. included in the present study. Unlike other cultivars, there are three types of chromosomes in the complement. Morphologically also it stands apart in having highly unlobed large leaves and long petiole.

Previous and present reports on chromosome number and cytology show the presence of $2 n=28$ chromosomes in most of the cultivars in this genus. Polyploidy, especially aneuploidy seems to have played a major role in the evolution of various taxa as evidenced by the occurrence of aneuploids with $2 \mathrm{n}=26,27,30$ chromosomes (Sinoto 1929, Das 1961, Kundu and Sharma 1976). The reason attributed by Kundu and Sharma (1976) for such an aneuploid occurrence is probably due to extensive vegetative propogation. No doubt, vegetative propagation has helped for the perpetuation of aneuploids rather than their origin. From the present work it may be concluded that outbreeding has played an important role in the origin of aneuploids due to the formation of gamete with unbalanced chromosome numbers. Occurrence of good number of natural triploids in Japan has been attributed to the process of fertilization between an unreduced female gamete and reduced pollen by Seki (1961). In all the above cases the cytological identity of each cultivar has been represented in phenotypic variation. However, extensive karyomorphological investigations coupled with meiotic behaviour, biochemical and physiological information seems to be imperative in drawing a conclusion about the taxonomical position of the taxa and the work in this direction has been in progress in this Institute.

\section{Summary}

Karyotype studies of ten exotic cultivars namely, Kosen, Kokuso-13, Kokuso-21, Kokuso27, Roso and Rokokuyso belonging to Morus latifolia Poir., Kenmochi, Mizusawa and Ichihei belonging to Morus bombycis Koidz. and Chinapeking belonging to $M$. alba $\mathrm{L}$. were reported for the first time. Of the ten cultivars, five are diploids $(2 n=28)$, two are triploids $(2 n=3 x=$ 42) and remaining three are aneuploids $(2 n=28+2)$. Chromosomes are generally small with an average length of $1.16 \mu$ and length ranges from $0.62 \mu$ to $1.70 \mu$ in all the cultivars 
studied. Relative length ranges from 96 to $131 \mu$. In most of the cases, complement consists of only two types of chromosomes with median and submedian centromeres resulting in bimodal karyotypes. As a whole, frequency of median chromosomes is more than submedian ones. Of the ten strains, two strains namely Kokuso-13 and Mizusawa bear satellite chromosomes. Morphological variation and evolution of taxa were attributed to structural changes in the karyotype and an attempt has been made to study the phylogenetic relationship among the varieties of each species based on their karyotype. Vegetative propogation seems to have played a major role in maintaining the taxa at different ploidy level.

\section{References}

Baker, H. C. 1970. Taxonomy and biological species concept in cultivated plants. In Genetic Resources in Plants, their Exploration and Conservation. Eds. O. H. Frankel and E. Bennett. Blackwell Scientific Publications, Oxford and Edinburgh, 49: 68.

Chennaveeraiah, M. S. 1983. Biosystamatics-A Presedential Address. 70th Session. Indian Science Congress, Section of Botany

Clausen, J., Keck D. D. and Hiesey, W. M. 1939. Amer. Jour. Bot. 26: 103-106.

Das, B. C. 1961. Cytological studies in Morus indica L. and Morus levigata Wall. Caryologia 14: 159-162.

- 1963. Polysomaty in mulberry. Sci. and Cult. 29: 250-252.

Datta, M. 1954. Cytogenetical studies in the species of Morus. Cytologia. 19: 86-95.

Gill, B. S. and Gupta, R. C. 1978. Cytological studies in the sex types of Morus alba L., Moraceae. Curr. Sci. 48: 35-36.

Hotta, T. 1953. Taxonomical study on the wild mulberry in Japan and near countries. Bot. Mag. Tokyo 66: $177-180$.

Janaki Ammal, E. K. 1948. The origin of black mulberry. J. Roy. Hort. Sci. 73: 117-120.

Katsumata, F. 1972. Mulberry species in Java and their pecularities. J. Seric. Sci. Japan. 41: 213-222.

- 1979. Chromosomes of Morus nigra L. from Java and hybridization affinity between this species and some mulberry species in Japan. J. Seric. Sci. Japan. 48 (3): 418-422.

Koidzumi, . 1917. Kawazokushakubatsuko. Bull. Imp. Seric. Exp. Sta. III: 1-PI-XI.

Kundu, D. and Sharma, A. 1976. Chromosome studies in some Indian Moraceae. In Recent Advances in Botany, edited by P. Kuchroo, Bishen Singh and Mahendrapal Singh, Dehradun. India. pp. 348-365.

Levan, A., Fredga, K. and Sandberg, A. A. 1964. Nomenclature for centromeric position on chromosome. Hereditas 51 : 201-220.

Mayer, E. 1942. Systematics and origin of species. Columbia University Press, New York.

Osawa, I. 1920. Cytological and experimental studies in Morus with special reference to triploid mutants. Bull. Imp. Seric. Exp. Stn. 1: 318-369.

Seki, H. 1952. Cytological studies in Moraceae (3) The triploid race. J. Seric. Sci. Japan 21: 211-214.

- 1961. Cytological studies on Morus Part-I. Polyploidy of mulberry tree with special reference to spontaneous occurrence of triploid plant. J. Fac. Tech. Sci. Sinshu Univ. 20: 1-59.

Sinoto, Y. 1929. Chromosome studies in some dioecious plants with special reference to allosomes. Cytologia 1: 109-144.

Tahara, M. 1909. On the chromosome of Morus alba (P. N.). Bot. Mag. Tokyo 23: 343-353. 